sities (WMH) and total brain volume (TBV). Methods: MRI quantification of brain and WMH volumes were obtained from 1974 Framingham Heart Study Offspring Cohort participants free from clinical stroke, TIA or dementia with an age range of $35-85$ years $(60 \pm 9 ; 53 \%$ women). To adjust for head size, both measures were divided by total cranial volume (TCV); WMH was $\log$ transformed to normalize variance. MRI measures were associated with 8 inflammatory biomarkers including CD40 ligand (CD40L), C-reactive protein (CRP), interleukin-6 (IL-6), soluble intracellular adhesion molecule-1 (sICAM-1), monocyte chemoattractant protein-1 (MCP-1), myeloperoxidase (MPO), osteoprotegerin (OPG), and P-selectin after log transformation, adjusting for age, sex, systolic blood pressure (BP), diastolic BP, body mass index, height, total high density lipoprotein, smoking status, fasting glucose, triglycerides, diabetes, BP treatment, hormone replacement therapy, lipid lowering treatment, aspirin, atrial fibrillation, prevalent CVD, and ECG left ventricular hypertrophy. Results: In multivariable models, the inflammatory markers were associated with TBV $(\mathrm{p}<.0001)$ but not WMH. Backward elimination (terms with $\mathrm{p}<0.05$ retained) revealed two significant inverse associations with TBV including IL-6 $(\beta=-0.29, p=.002)$ and OPG $(\beta=-0.77, p=.001)$. The estimated mean change in TBV per interquartile range of IL- $6=-0.26(p=.002)$ and $\mathrm{OPG}=-0.30(\mathrm{p}=.001)$. Findings were similar when the analyses were re-run excluding individuals with prevalent CVD. Conclusions: Our analyses revealed that IL-6 and OPG, both markers of systemic inflammation, were inversely associated with $\mathrm{TBV}$; however, no inflammatory marker was associated with WMH. Though our analyses are cross-sectional and unable to establish causality, we propose that elevated inflammatory markers may be implicated in accelerating age-related atrophy. The etiology of this association remains unclear and requires further investigation.

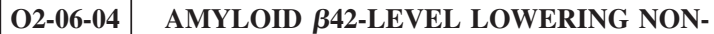 STEROIDAL ANTI-INFLAMMATORY DRUGS AND THE RISK OF ALZHEIMER'S DISEASE}

Mendel D. Haag ${ }^{1}$, Marieke van Oijen ${ }^{1,2}$, Frank Jan de Jong ${ }^{1,2}$, Albert Hofman ${ }^{1}$, Theo Stijnen ${ }^{1}$, Bruno H. Stricker ${ }^{1,3}$,

Monique M. Breteler ${ }^{1},{ }^{l}$ Department of Epidemiology \& Biostatistics, Erasmus Medical Center, Rotterdam, The Netherlands; ${ }^{2}$ Department of Neurology, Erasmus Medical Center, Rotterdam, The Netherlands; ${ }^{3}$ Inspectorate for Health Care, The Hague, The Netherlands. Contact e-mail:m.haag@erasmusmc.nl

Background: Initial interest in non-steroidal anti-inflammatory drugs (NSAIDs) for prevention of Alzheimer's disease (AD) was driven by evidence of inflammation in AD pathology. We previously demonstrated that long-term use of NSAIDs was associated with a decreased risk of AD. Results from in vitro and in vivo studies now suggest that a protective effect of NSAIDs may be mediated by their effect on amyloid- $\beta 42$ (A $\beta 42)$ levels, rather than by anti-inflammatory activity. Not all NSAIDs lower A $\beta 42$ levels. Objective: To determine whether use of A $\beta 42$-lowering NSAIDs is associated with a lower risk of AD. Methods: The study population consisted of 6992 participants of the prospective, populationbased Rotterdam Study, who were 55 years or older and free of dementia at baseline. Complete information on filled prescriptions was obtained from automated pharmacy records. NSAIDs were classified as A $\beta 42$-lowering or non-A $\beta 42$-lowering agents. We defined four mutually exclusive timedependent categories of cumulative NSAID use: no use, short-term $(\leq 1$ month), intermediate-term ( 1 to 24 months) and long-term use ( $\geq 24$ months). The reference group for all analyses was no use of any NSAID. Data were analysed with Cox proportional-hazard models, adjusting for age, sex, concomitant cumulative use of other NSAIDs, education, diabetes mellitus and cumulative use of oral anticoagulants and antihypertensives. In addition, we stratified on APOE genotype. Results: During follow-up (mean 9.0 years) 739 persons developed dementia, of whom 582 developed $\mathrm{AD}, 81$ vascular dementia and 76 other types of dementia. Long-term use of any NSAID was associated with a non-significant risk reduction of AD (RR 0.65 ; 95\% confidence interval (CI); 0.40-1.06) compared to no use of any NSAID. Long-term use of A $\beta 42$-lowering NSAIDs was associated with a significantly decreased risk of AD (RR 0.42 ; 95\% CI $0.20-0.90$ ) compared to no use of any NSAID, whereas use of non-A $\beta 42$-lowering NSAIDs was not associated with a decreased risk of AD (RR 0.78; 95\%CI 0.32-1.90). Relative risks were lower in presence of the APOE4 allele, but interactions were not significant. Conclusions: Long-term use of A $\beta 42-$ lowering NSAIDs seems to protect against AD. These findings are in agreement with the effects of NSAIDs on A $\beta 42$ levels observed in vitro and in vivo.

\section{2-06-05 HOMOCYSTEINE AND INCIDENCE OF DEMENTIA AND COGNITIVE IMPAIRMENT IN OLDER MEXICAN AMERICANS: RESULTS FROM THE SACRAMENTO AREA LATINO STUDY ON AGING}

Mary N. Haan ${ }^{1}$, William J. Jagust ${ }^{2}$, Josh W. Miller ${ }^{3}$, Allilson A. Aiello ${ }^{1}$, Dan M. Mungas ${ }^{3}$, Lindsay H. Allen ${ }^{3}$, Ralph R. Green ${ }^{3},{ }^{1}$ University of Michigan, Ann Arbor, MI, USA; ${ }^{2}$ University of California, Berkeley, CA, USA; ${ }^{3}$ University of California, Davis, CA, USA. Contact e-mail: mnhaan@umich.edu

Background: Although cereals and flours are universally fortified with folic acid, recent epidemiologic studies have linked elevated homocysteine (HCY) to increased risk of Alzheimer's, dementia and cognitive decline and underlying brain pathology. Reduction of HCY by B vitamin supplementation inspires investigation of the role of HCY in dementias. Objective: The associations of homocysteine, erythrocyte folate, and plasma B12 with 4.5 year incidence of dementia and cognitive impairment not dementia (CIND) were examined in a cohort of 1,789 Mexican Americans begun in 1998-99. Methods: Dementia and CIND $(n=102)$ were ascertained in 2000-2005 by neuropsychological and clinical exams and expert adjudication. The association between HCY and dementia/CIND incidence was examined using a series of proportional hazards models. HCY, B12 and folate were log-transformed. A subset of 172 participants had volumetric MRIs. White matter (WMSH) was quantified using standard methods. The influence of HCY on WMSH (log) was examined using multivariate linear regression. Results: HCY $(\log )$ was associated with an increased risk of dementia/CIND: (HR: 2.13, 95\% CI: 1.07-4.44). Adjustment for plasma $\mathrm{B} 12$, red blood cell (RBC) folate, renal function, stroke, age, education, gender, country of birth, and vitamin use did not influence this association. $\mathrm{RBC}$ folate was not associated with dementia/CIND but low plasma B12 was associated with a slightly increased risk (HR: $1.05 .95 \%$ CI 1.01-1.09). Baseline stroke was associated with a nearly 2.5 -fold increased risk (HR: 2.44: 95\% CI: 1.37-4.34). Exclusion of participants with stroke at baseline did not affect the associations of HCY and dementia/CIND. In the bottom two tertiles of $\mathrm{B} 12(\leq 500 \mathrm{pg} / \mathrm{mL})$, $\mathrm{HCY}$ was associated with an increased risk of dementia/CIND (HR: 2.03, $\mathrm{p}=0.007$ ) but not in the top tertile of B12 (HR: 1.10, $\mathrm{p}=0.90)$. HCY was associated with increased WMSH (age adjusted): $(\beta=0.62, p=.04)$. Conclusions: Even in the presence of universal folic acid fortification of food, HCY remains a significant risk factor for dementia and cognitive impairment. B12 supplementation may reduce the effects of HCY on dementia risk. However, the influence of HCY on dementia does not depend entirely on B vitamin levels. The link between HCY and WMSH suggests vascular pathology may be involved.

\section{O2-06-06 THE ASSOCIATION OF CHOLESTEROL WITH DEMENTIA OVER TIME IN THE PROSPECTIVE POPULATION STUDY OF WOMEN IN GOTHENBURG, SWEDEN}

Peter P. Zandi ${ }^{1}$, Michelle M. Mielke ${ }^{1}$, Huibo $\mathrm{Shao}^{1}$, Xinxin $\mathrm{Guo}^{2}$, Margda Waern ${ }^{2}$, Simona Sacuiu ${ }^{2}$, Svante Ostling ${ }^{2}$, Deborah Gustafson ${ }^{3}$, Ingmar Skoog $2,{ }^{1}$ Johns Hopkins University, Baltimore, MD, USA; ${ }^{2}$ Gothenburg University, Gothenburg, Sweden; ${ }^{3}$ Gothenburg University, Gothenburg, Sweden. Contact e-mail: pzandi@jhsph.edu

Background: There are conflicting reports on the relationship between cholesterol and dementia. Several studies suggest that high cholesterol is a risk 